\title{
EXISTENCE AND DECAY OF SOLUTIONS OF SOME NONLINEAR PARABOLIC VARIATIONAL INEQUALITIES
}

\author{
MITSUHIRO NAKAO \\ Department of Mathematics \\ College of General Education \\ Kyushu University \\ Fukuoka, Japan \\ TAKASHI NARAZAKI \\ Department of Mathematical Sciences \\ Tokai University \\ Kanagawa, Japan \\ (Recelved January 23, 1979)
}

ABSTRACT. This paper discusses the existence and decay of solutions $u(t)$ of the variational inequality of parabolic type:

$$
<u^{\prime}(t)+A u(t)+B u(t)-f(t), v(t)-u(t)>\geq 0
$$

for $\forall v \in L^{P}([0, \infty) ; V \quad(p \geq 2)$ with $v(t) \in K$ a.e. in $[0, \infty)$, where $K$ is a closed convex set of a separable uniformly convex Banach space V, A is a nonlinear monotone operator from $\mathrm{V}$ to $\mathrm{V} *$ and $\mathrm{B}$ is a nonlinear operator from Banach space $\mathrm{W}$ to $\mathrm{W}^{*} . \mathrm{V}$ and $\mathrm{W}$ are related as $\mathrm{V} \subset \mathrm{W} \subset \mathrm{H}$ for a Hilbert space $\mathrm{H}$. No monotonicity assumption is made on $B$.

KEY WORDS AND PHRASES. Existence, Decay, Nonlinear parabolic variational inequalities. 1980 MATHEMATICS SUBJECT CLASSIFICATION CODES. 35K55. 


\section{Introduction}

Let $\mathrm{H}$ be a real Hilbert space with norm $\mid, V$ be a real separable uniformly convex Banach space with norm \|\|$_{V}$ densely imbedded in $H$ and let $K$ be a closed convex subset of $\mathrm{V}$ containing 0 . Moreover, let $\mathrm{W}$ be a Banach space with norm \|\|$_{W}$ such that $\mathrm{V}$ (W (H. We suppose that the natural injections from $V$ into $W$ and from $W$ into $H$ are compact and continuous, respectively. We identify $\mathrm{H}$ with its dual space $\mathrm{H}^{*}$ (i.e., $\mathrm{V}\left(\mathrm{W}\left(\mathrm{H}\left(\mathrm{W}^{*}\left(\mathrm{~V}^{*}\right)\right.\right.\right.$. Pairing between $\mathrm{V}^{*}$ and $\mathrm{V}$ will be denoted by $\left\langle\mathrm{v}^{*}, \mathrm{v}\right\rangle$ for $\mathrm{v}^{*} \in \mathrm{V}^{*}$ and $\mathrm{v} \in \mathrm{V}$. Consider the following variational inequality of parabolic type :

$$
\left\langle u^{\prime}(t)+A u(t)+B u(t)-f(t), v(t)-u(t)\right\rangle \geqq 0
$$

for $v(t) \in L^{p}([0, \infty) ; v) \quad(p \geq 2)$ with $v(t) \in K$ a.e. in $(0, \infty)$. A solution $u(t)$ of ( 1 ) should satisfy the conditions : $u(t) \in L_{\text {loc }}^{p}([0, \infty) ; v) \bigcap c([0, \infty) ; H), u^{\prime}(t) \in L_{l O C}^{2}([0, \infty) ; H)$,

$u(t) \in K$ for a.e. $t \in[0, \infty)$ and the initial condition

$$
u(0)=u_{0} \epsilon K
$$

Here $A$ is a monotone operator from $V$ to $V^{*}$ and $B$ is a bounded operator from $W$ to $W^{*}$. More precisely we make the following assumptions on them. 
$A_{1}$. $A$ is the Fréchet derivative of a convex functional $F_{A}(u)$ on $\mathrm{V}$, hemicontinuous on $\mathrm{V}$ and satisfies the inequalities

$$
\mathrm{k}_{0}\|\mathrm{u}\|_{\mathrm{V}}^{\mathrm{p}} \leqq \mathrm{F}_{\mathrm{A}}(\mathrm{u}) \quad(\leqq<\mathrm{Au}, \mathrm{u}>)
$$

with some $k_{0}>0$ and $p \geq 2$, and

$$
\|A u\|_{V^{*}} \leq C_{0}\left(\|u\|_{V}\right)
$$

where $C_{0}(\cdot)$ is a monotone increasing function on $[0, \infty)$.

$A_{2}$. $B$ is the Frechet derivative of a functional $F_{B}(u)$ on $w$, continuous on $W$ and satisfies

$$
\|\mathrm{Bu}\|_{\mathrm{W}^{*}} \leq \mathrm{k}_{1}\|\mathrm{u}\|_{\mathrm{W}}^{\alpha+1}
$$

with some $k_{1}, \alpha>0$.

Regarding the forcing term $f(t)$ we assume :

$A_{3} . \quad f \in L_{1 \text { oc }}^{q}\left([0, \infty) ; V^{*}\right) \bigcap L_{10 c}^{2}([0, \infty) ; H)$ with $q=p /(p-1)$ and

$$
\begin{aligned}
\delta(t) & \equiv \max \left\{\left(\int_{t}^{t+1}\|f(s)\|_{\mathrm{V}^{*}}^{\mathrm{q}} \mathrm{ds}\right)^{1 / \mathrm{q}},\left(\int_{t}^{t+1}|f(s)|^{2} \mathrm{ds}\right)^{1 / 2}\right\} \\
& \leqq \text { const. }<\infty .
\end{aligned}
$$

Note that no monotonicity condition on $B$ is assumed. The problem ( 1 ) is said 'unperturbed' if $B(t) \equiv 0$, and said 'perturbed' if $B(t) \not \equiv 0$. The unperturbed problem (1) with the initial condition (2) is familiar, and the existence and unique- 
ness theorems are known in more general situations than ours (see Lions [5], Brezis [2], Biroli [1], Kenmochi [4], Yamada [13], etc.). However the asymptotic behaviors of solutions as $t \rightarrow \infty$ seem to be known little. In this note we first prove a decay property of solutions of the unperturbed problem (1)-(2) (with $B(t) \equiv 0)$. This result is derived by combining the penalty method with the argument in our previous paper [10], where the nonlinear evolution equations (not inequalities) were treated.

Next we consider the perturbed problem (1)-(2) (i.e., B(t) 70 ). For the equation $u^{\prime}(t)+A u(t)+B u(t)=f(t)$ (not inequality), the existence of bounded solutions on $[0, \infty)$ in the norm $\|_{V}$ was proved in [8] (see also [7]). We extend this result to the variational inequality (1)-(2). Recently, similar problems were treated by ôtani [12] and Ishii [3] in the framework of the theory of subdifferential operators. In their works it is assumed that $f(t) \equiv 0$ or $\int_{0}^{\infty}|f(s)|_{H}^{2} d s$ is small, while here we require only the smallness of $M \equiv \sup _{t} \delta(t)$. Ishii [3] discussed the decay or blowing up properties of solutions. We also prove a decay property of solutions of the perturbed problem. Our result is much better than the corresponding result of [3] .

We employ the so-called penalty method introduced by Lions [5], and the argument is related to the one used in our previous paper [1I], where the nonlinear wave equations in noncylindrical domains were considered. 
1. Preliminaries

We prepare some lemmas concerning a penalty functioanl $B(u)$. Let $K$ be a closed convex set in $V$ and let $J: V \rightarrow V^{*}$ be the duality mapping such that

$$
\|\mathrm{J}(\mathrm{u})\|_{\mathrm{V}^{*}}=\|\mathrm{u}\|_{\mathrm{V}},\langle\mathrm{J}(\mathrm{u}), \mathrm{u}\rangle=\|\mathrm{u}\|_{\mathrm{V}}^{2}
$$

Then the penalty functional $\beta(u)$ for $K$ is defined by

$$
\beta(u)=J\left(u-p_{K} u\right)
$$

where $\mathrm{p}_{\mathrm{K}}$ is the projection of $\mathrm{V}$ to $\mathrm{K}$. Recall that $\mathrm{p}_{\mathrm{K}} \mathrm{u}$ $(\in K)$ is determined by

$$
\left\|\mathrm{u}-\mathrm{p}_{\mathrm{K}} \mathrm{u}\right\|_{\mathrm{V}}=\underset{\mathrm{win} \in \mathrm{K}}{\min }\|\mathrm{w}\|_{\mathrm{V}}
$$

$\mathrm{p}_{\mathrm{K}} \mathrm{u}$ is also characterized as the unique element of $\mathrm{K}$ satisfying

$$
<J\left(u-p_{K} u\right), w-p_{K} u>\leqq 0 \quad \text { for } \quad w \in K \text {. }
$$

For a proof see Lions [5]. The following two lemmas are well known.

Lemma 1. (Lions [5])

$\beta(u)$ is a monotone hemicontinuous mapping from $V$ to $V^{*}$.

Lemma 2. (see, e.g., [6])

The projection $\mathrm{p}_{\mathrm{K}}$ is continuous. 
The next lemma plays an essential role in our arguments.

Lemma 3 .

Let $u(t) \in C^{l}([0, \infty) ; V)$. Then $\left\|u(t)-p_{K} u(t)\right\|_{V}^{2}$ is differentiable on $[0, \infty)$ and it holds that

$$
\frac{1}{2} \frac{d}{d t}\left\|u(t)-p_{K} u(t)\right\|_{V}^{2}=\left\langle\beta(u(t)), u^{\prime}(t)\right\rangle .
$$

Proof.

The proof can be given by a variant of the way in Biroli [1, lemma 6]. By a standard argument (see Lions [5, Chap II, Prof 8.1]) we know

$$
\frac{1}{2}\left\|\mathrm{w}-\mathrm{p}_{\mathrm{K}} \mathrm{w}\right\|_{\mathrm{V}}^{2}-\frac{1}{2}\left\|\mathrm{v}-\mathrm{p}_{\mathrm{K}} \mathrm{v}\right\|_{\mathrm{V}}^{2} \geqq\langle\beta(\mathrm{v}), \mathrm{w}-\mathrm{v}\rangle
$$

for $w, v \in V$. Then, if $t, t+h \geqslant 0$ we have

$$
\frac{1}{2}\left\|u(t+h)-p_{K} u(t+h)\right\|_{V}^{2}-\frac{1}{2}\left\|u(t)-p_{K} u(t)\right\|_{V}^{2}
$$

$$
\geqq\langle\beta(u(t)), u(t+h)-u(t)\rangle \text {. }
$$

If $h>0$, we have from (12)

$$
\frac{1}{2 h} \int_{t_{2}}^{t_{2}+h}\left\|u(s)-p_{K} u(s)\right\|_{V}^{2} d s-\frac{1}{2 h} \int_{t_{1}}^{t_{1}+h}\left\|u(s)-p_{K} u(s)\right\|_{V}^{2} d s
$$

$$
\geq \int_{t_{1}}^{t_{2}}<\beta(u(s)), \frac{u(s+h)-u(s)}{h}>d s
$$

for $t_{2}>t_{1} \geq 0$, and hence, letting $h \downarrow 0$, 


$$
\frac{1}{2}\left\|u\left(t_{2}\right)-p_{K} u\left(t_{2}\right)\right\|_{V}^{2}-\frac{1}{2}\left\|u\left(t_{1}\right)-p_{K} u\left(t_{1}\right)\right\|_{V}^{2}
$$

$$
\geqq \int_{t_{1}}^{t_{2}}<\beta(u(s)), u^{\prime}(s)>d s .
$$

Similarly, if $\mathrm{h}<0$, we have

$$
\begin{aligned}
& \frac{1}{2 h} \int_{t_{2}+h}^{t_{2}}\left\|u(s)-p_{K} u(s)\right\|_{V}^{2} d s-\frac{1}{2 h} \int_{t_{1}+h}^{t_{1}}\left\|u(s)-p_{K} u(s)\right\|_{V}^{2} d s \\
& \leq \int_{t_{1}}^{t_{2}}<\beta(u(s)), \frac{u(s+h)-u(s)}{h}>d s
\end{aligned}
$$

for $t_{2}>t_{1}$ with $t_{1}+h>0$, and

$$
\begin{aligned}
& \frac{1}{2}\left\|u\left(t_{2}\right)-p_{K} u\left(t_{2}\right)\right\|_{V}^{2}-\frac{1}{2}\left\|u\left(t_{1}\right)-p_{K} u\left(t_{1}\right)\right\|_{V}^{2} \\
& \leqq \int_{t_{1}}^{t_{2}}<B(u(s)), u^{\prime}(s)>d s
\end{aligned}
$$

for $t_{2}>t_{1} \geq 0$, where we have used the continuity of $p_{K} u(t)$ at $t \gg 0$. The inequalities (14) and (15) are equiavlent to (10).

We conclude this section by stating a lemma concerning a difference inequality, which will be used for the proof of decay of solutions.

Lemma 4 . ([9])

Let $\phi(t)$ be a nonnegative function on $[0, \infty)$ such that

$$
\sup _{t \leqq S \leq t+1} \phi(t)^{1+r} \leqq C_{0}(\phi(t)-\phi(t+1))+g(t)
$$


with some $C_{0}>0$ and $r \geq 0$. Then

(i) if $r=0$ and $g(t) \leqq C_{1} \exp (-\lambda t)$ with some $\lambda>0, c_{1}>0$, then $\phi(t) \leq C_{i}^{\prime} \exp \left(-\lambda^{\prime} t\right)$ for some $C_{i}^{\prime}, \lambda^{\prime}>0$,

and

(ii) if $r>0$ and $\lim _{t \rightarrow \infty} g(t) t^{1+1 / r}=0$, then

$$
\phi(t) \leqq C_{1}^{\prime}(1+t)^{-1 / r} \quad \text { for some } C_{1}^{\prime}>0 .
$$

2. Unperturbed problem

As is mentioned in the introduction we prove here a decay property of solutions of the unperturbed problem (1)-(2).

Theorem 1 .

Let $u_{0} \in K$ and let $\lim _{t \rightarrow \infty} \delta(t) t^{(p-1) /(p-2)}=0$ if $p>2$ and $\delta(t) \leqq C \exp (-\lambda t) \quad(\lambda>0)$ if $p=2$. Then the problem (1)-(2) with $B(t) \equiv 0$ admits a unique solution $u(t)$, satisfying

$$
\|u(t)\|_{V} \leq c\left(\left\|u_{0}\right\|_{V}\right)(1+t)^{-1 /(p-2)} \text { if } p>2
$$

and

$$
\|u(t) \quad\|_{V} \leq c\left(\left\|u_{0}\right\|_{V}\right) \exp (-\lambda \cdot t) \quad \text { if } p=2
$$

with some $\lambda^{\prime}>0$. 
Proof.

Recall that the solution $u$ is given by a limit function of $\left\{u_{\varepsilon}(t)\right\}$ as $\varepsilon \longrightarrow 0$, where $u_{\varepsilon}(t)$ is the solution of the modified equation

(17)

$$
\left\{\begin{array}{l}
u^{\prime}(t)+A u(t)+\frac{1}{\varepsilon} \beta(u)=f(t) \quad(\varepsilon>0) \\
u(0)=u_{0} .
\end{array}\right.
$$

Since $A$ and $B$ are monotone hemicontinuous operators from $V$ to $V^{*}$, the problem (16) has a unique solution $u_{\varepsilon}(t)$ such that

$$
u_{\varepsilon}(t) \in L_{I O C}^{p}([0, \infty) ; V) \text { and } u_{\varepsilon}^{\prime}(t) \in L_{l o c}^{2}([0, \infty) ; H) .
$$

(Cf. Lions [5, Chap. 2, Th. 1.2., see also Biroli [1], where more general result is given.)

Let $\left\{w_{j}\right\}_{j=1}^{\infty}$ be a basis of $v$. Then, it is known that $u_{\varepsilon}(t)$ is given by the limit function of $\left\{u_{m, \varepsilon}(t)\right\}$ as $m \rightarrow \infty$, where $u_{m, \varepsilon}(t)=\sum_{j=1}^{m} \alpha_{j, m}(t) w_{j}$ is the solution of

$$
\begin{aligned}
& \left\langle u_{m, \varepsilon}^{\prime}(t), w_{j}\right\rangle+\left\langle A u_{m, \varepsilon}(t), w_{j}\right\rangle=\left\langle f(t), w_{j}\right\rangle \\
& (j=1,2, \ldots, m)
\end{aligned}
$$

with the initial condition

$$
u_{m, \varepsilon}(0)=u_{m, \varepsilon}^{0} \longrightarrow u_{0} \text { in } v \text {. }
$$


The problem (17)-(18) is a system of ordinary differential equations with respect to $\alpha_{j, m}(t), j=1,2, \ldots, m$, and by the monotonicity and hemicontinuity of $A$ and $\beta$ it is easy to see that this problem admits unique solution such that

$$
u_{m, \varepsilon}(t) \in C^{1}\left([0, \infty) ; v_{m}\right) \subset c^{1}([0, \infty) ; v)
$$

where $v_{m}$ is the m-dimensional subspace of $V$ spanned by $\left\{w_{1}\right.$, $\ldots, n_{m}$. For the proof of Theorem 1 , it suffices to show that the estinate (16) or (16)' with $u=u_{m, \varepsilon}$ holds with the constants independent of $m$ and $\varepsilon$.

By Lemma 3 we have

$$
\begin{aligned}
& E_{\varepsilon}\left(u_{m, \varepsilon}\left(t_{2}\right)\right)-E_{\varepsilon}\left(u_{m, \varepsilon}\left(t_{1}\right)\right)+\int_{t_{1}}^{t_{2}}\left|u_{m, \varepsilon}^{\prime}(s)\right|^{2} d s \\
& =\int_{t_{1}}^{t_{2}}<f(s), u_{m, \varepsilon}^{\prime}(s)>d s
\end{aligned}
$$

for $t_{2}>t_{1} \geq 0$, where

$$
E_{\varepsilon}(u(t)) \equiv F_{A}(u(t))+\frac{1}{2 \varepsilon}\left\|u(t)-p_{K} u(t)\right\|_{V}^{2} .
$$

Also we have easily by (18)

$$
\begin{aligned}
& \int_{t_{1}}^{t_{2}}\left\{<A u_{m, \varepsilon}(s), u_{m, \varepsilon}(s)>+\frac{1}{\varepsilon}<\beta\left(u_{m, \varepsilon}(s), u_{m, \varepsilon}(s)>\right\} d s\right. \\
& (21) \quad=\int_{t_{1}}^{t}\left\{\left\langle f(s), u_{m, \varepsilon}(s)>-<u_{m, \varepsilon}^{\prime}(s), u_{m, \varepsilon}(s)>\right\} d s .\right.
\end{aligned}
$$

Using the similar argument as in [10], the equalities (20)-(21) 
imply the estimate (16) or (16)' with $u=u_{m, \varepsilon}$. For completeness, however, we sketch the proof briefly.

By (20) we have

$$
\int_{t}^{t+1}\left|u_{m, \varepsilon}^{\prime}(s)\right|^{2} d s \leqq 2\left\{E_{\varepsilon}\left(u_{m, \varepsilon}(t)-E_{\varepsilon}\left(u_{m, \varepsilon}(t+1)\right)\right\}+C \delta(t)\right.
$$

(22)

$$
\equiv D_{\varepsilon}(t)^{2} \cdot \quad(C>0 ; \text { constant })
$$

On the other hand, using the ineqaulity

$$
\begin{aligned}
& \left.<A u_{m, \varepsilon}(t), u_{m, \varepsilon}(t)\right\rangle+\frac{1}{\varepsilon}\left\langle\beta\left(u_{m, \varepsilon}(t)\right), u_{m, \varepsilon}(t)>\geqq E_{\varepsilon}\left(u_{m, \varepsilon}(t)\right)\right. \\
& (\text { see (3) and (9)), }
\end{aligned}
$$

we have from (21)

$$
\begin{aligned}
\int_{t}^{t+1} E_{\varepsilon}\left(u_{m, \varepsilon}(s)\right) d s \leqq & \left(\int_{t}^{t+1}\|f(s)\|_{V^{*}}^{2} d s\right)^{1 / 2} \sup _{s \in[t, t+1]}\left\|u_{m, \varepsilon}(s)\right\|_{V} \\
& +\left(\int_{t}^{t+1}\left|u_{m, \varepsilon}^{\prime}(s)\right|^{2} d s\right)^{1 / 2} \sup _{t \leqq s \leqq t+1}\left|u_{m, \varepsilon}(s)\right|
\end{aligned}
$$

$$
\leqq C\left(D_{\varepsilon}(t)+\delta(t) \sup _{t \leqq s \leqq t+1} E_{\varepsilon}\left(u_{m, \varepsilon}(s)\right)^{1 / p}\right.
$$

where hearafter $C$ denotes various constants independent of $m$ and $\varepsilon$. From (23) there exists $t^{*} \in[t, t+1]$ such that

$$
\left.E_{\varepsilon}\left(u_{m, \varepsilon}\left(t^{*}\right)\right) \leq C\left\{D_{\varepsilon}(t)+\delta(t)\right)\right\} \sup _{t \leq s \leq t+1} E_{\varepsilon}\left(u_{m, \varepsilon}(s)\right)^{1 / p}
$$

and hence by $(20)$ 


$$
\begin{aligned}
\sup _{t \leq s \leq t+1} E_{\varepsilon}\left(u_{m, \varepsilon}(s)\right) \leq C\left\{\left(D_{\varepsilon}(t)+\delta(t)\right)\right. & \sup _{t \leq s \leq t+1_{\varepsilon}} E_{\varepsilon}\left(u_{m, \varepsilon}(s)\right)^{1 / p} \\
& \left.+D_{\varepsilon}(t)^{2}+D_{\varepsilon}(t) \delta(t)\right\}
\end{aligned}
$$

and by Young's inequality,

$$
\begin{aligned}
\sup _{t \leq s \leq t+1} E_{\varepsilon}\left(u_{m, \varepsilon}(s)\right) \leqq & c\left\{\left(D_{\varepsilon}(t)+\delta(t)\right) p /(p-1)\right. \\
& \left.+D_{\varepsilon}(t)^{2}+\delta(t)^{2}\right\} .
\end{aligned}
$$

From (24) we can easily see that $E_{\varepsilon}\left(u_{m, \varepsilon}(t)\right)$ is bounded on $[0, \infty)$ by a constant depending on $E_{\varepsilon}\left(u_{m, \varepsilon}(0)\right)$. Since we may assume, without loss of generality, that $u_{m, \varepsilon}(0) \in K$ and

$$
E_{\varepsilon}\left(u_{m, \varepsilon}(t)\right) \leqq C\left(E_{\varepsilon}\left(u_{m, \varepsilon}(0)\right)\right) \leqq C\left(\left\|u_{0}\right\|_{V}\right)
$$

where $C(\cdot)$ denotes various constants depending on the indicated quantity. By $(20)$ and (25) we have

$$
\begin{aligned}
& \sup _{t \leq s \leq t+1} E_{\varepsilon}\left(u_{m, \varepsilon}(s)\right)^{2(p-1) / p} \\
& \leq C\left(\left\|u_{0}\right\|_{V}, M\right)\left\{E_{\varepsilon}\left(u_{m, \varepsilon}(t+1)\right)-E_{\varepsilon}\left(u_{m, \varepsilon}(t)\right)+\delta(t)^{2}\right\}
\end{aligned}
$$

where we set MEsup $\delta(t)^{2}$. Applying Lemma 4 we obtain the desired result. 


\section{Perturbed problem}

In this section we investigate the existence and decay of solutions of the problem (1)-(2) with B satisfying the assumption $A_{2}$. For this consider the approximate equations

(27) $<u_{m, \varepsilon}^{\prime}(t)+A u_{m, \varepsilon}(t)+B u_{m, \varepsilon}(t)+\frac{1}{\varepsilon} \beta\left(u_{m, \varepsilon}(t)\right)-f(t), w_{j}>=0$,

$j=1,2, \ldots, m$, where we set again

$$
u_{m, \varepsilon}(t)=\sum_{j=1}^{m} a_{m, j}(t) w_{j} .
$$

and we impose $u_{m, \varepsilon}(0) \in K$ and $u_{m, \varepsilon}(0) \rightarrow u_{0}(\in K)$ in $v$. Using a similar argument as in [7] we derive a priori estimates for $u_{m, \varepsilon}(t)$. We also give a rather brief discussion. First we assume $p>\alpha+2$. By (27) we have

$$
\begin{aligned}
& G_{\varepsilon, 0}\left(u_{m, \varepsilon}\left(t_{2}\right)\right)-G_{\varepsilon, 0}\left(u_{m, \varepsilon}^{\prime}\left(t_{1}\right)\right)+\int_{t_{1}}^{t_{2}}\left|u_{m, \varepsilon}^{\prime}(s)\right|^{2} d s \\
& =\int_{t_{1}}^{t_{2}}<f(s), u_{m, \varepsilon}^{\prime}(s)>d s
\end{aligned}
$$

where

$$
G_{\varepsilon, 0}(u(t))=F_{A}(u(t))+F_{B}(u(t))+\frac{1}{2 \varepsilon}\left\|u(t)-p_{K} u(t)\right\|_{V}^{2} \text {, }
$$

and hence, in particular,

$$
G_{\varepsilon, 0}\left(u_{m, \varepsilon}(t)\right) \leqq G_{\varepsilon, 0}\left(u_{m, \varepsilon}(0)\right)+\frac{1}{4} \delta(0) \text { if } 0 \leqq t<1
$$


which together with the assumption $p>\alpha+2$ implies

$$
\left\|u_{m, \varepsilon}(t)\right\|_{V} \leqq c\left(\left\|u_{0}\right\|_{V}, \delta(0)\right)<\infty
$$

if $0 \leq t<1$. Thus $u_{m, \varepsilon}(t)$ exists on an interval, say $\left[0, t_{m}\right]$, with $t_{m}>1$. If we assume $G_{\varepsilon, 0}\left(u_{m, \varepsilon}(t)\right) \leqq G_{\varepsilon, 0}\left(u_{m, \varepsilon}(t+1)\right)$ for some $t>0$, we have from (28)

$$
\int_{t}^{t+1}\left|u_{m, \varepsilon}^{\prime}(s)\right|^{2} d s \leqq \delta(t)^{2} \leq M^{2}
$$

Using (27) and (31) we have

$$
\int_{t}^{t+1} G_{\varepsilon, l}\left(u_{m, \varepsilon}(t)\right) d s \leqq M^{2}+c \int_{t}^{t+1}\left\|u_{m, \varepsilon}(s)\right\|_{V}^{2} d s
$$

where we set

$$
G_{\varepsilon, 1}(u)=\left\langle A u+B u+\frac{1}{\varepsilon} \beta(u), u\right\rangle .
$$

Since

$$
\mathrm{G}_{\varepsilon, 1}(\mathrm{u}) \geqq \mathrm{k}_{0}\|\mathrm{u}\|_{\mathrm{V}}^{\mathrm{p}}-\mathrm{k}_{1}\|\mathrm{u}\|_{\mathrm{W}}^{\alpha+2}+\frac{1}{\varepsilon}\left\|\mathrm{u}-\mathrm{p}_{\mathrm{K}} \mathrm{u}\right\|_{\mathrm{V}}^{2}
$$

and since $p>\alpha+2$, therexists a point $t^{*} \in[t, t+1]$ such that

$$
\left\|u_{m, \varepsilon}\left(t^{*}\right)\right\|_{V}+\frac{1}{\varepsilon}\left\|u_{m, \varepsilon}\left(t^{*}\right)-p_{K} u_{m, \varepsilon}\left(t^{*}\right)\right\|_{V}^{2} \leq c(M) .
$$

From this and (28) 


$$
G_{\varepsilon, 0}\left(u_{m, \varepsilon}(t+1)\right) \leqq G_{\varepsilon, 0}\left(u_{m, \varepsilon}\left(t^{*}\right)\right)+C \delta(t)^{2} \leqq C(M) .
$$

Thus we conclude that

$$
\begin{aligned}
G_{\varepsilon, 0}\left(u_{m, \varepsilon}(t)\right) & \leqq \max \left(C(M), \max _{0 \leq s \leq 1} G_{\varepsilon, 0}\left(u_{m, \varepsilon}(s)\right)\right) \\
& \leqq C\left(M,\left\|u_{0}\right\|_{V}\right) \quad(\text { by }(29))
\end{aligned}
$$

and therefore $u_{m, \varepsilon}(t)$ exists on $[0, \infty)$, satisfying (32) $\quad\left\|u_{m, \varepsilon}(t)\right\|_{V}+\frac{1}{\varepsilon}\left\|u_{m, \varepsilon}(t)-p_{K} u_{m, \varepsilon}(t)\right\|_{V}^{2} \leqq c\left(M,\left\|u_{0}\right\|_{V}\right)$.

of course we know

$$
\int_{t}^{t+1}\left|u_{m, \varepsilon}^{\prime}(s)\right|^{2} d s \leqq c\left(M,\left\|u_{0}\right\|_{V}\right) \text { for } t \geqq 0
$$

We have now derived a priori estimate for $u_{m, \varepsilon}(t)$. Using standard compactness and monotonicity arguments (see Lions [5], Biroli [1] etc.) we can suppose without loss of generality that as $\mathrm{m} \longrightarrow \infty$,

$$
\begin{aligned}
& u_{m, \varepsilon}(t) \longrightarrow u_{\varepsilon}(t) \text { weakly* in } L^{\infty}([0, \infty) ; V), \\
& u_{m, \varepsilon}^{\prime}(t) \longrightarrow u_{\varepsilon}^{\prime}(t) \text { weakly in } L_{l o c}^{2}([0, \infty) ; V) .
\end{aligned}
$$

(34) $A u_{m, \varepsilon}(t)+\frac{1}{\varepsilon} \beta\left(u_{m, \varepsilon}(t)\right) \rightarrow X_{\varepsilon}(t)$ weakly** in $L^{\infty}\left([0, \infty) ; V^{*}\right)$,

$$
\mathrm{Bu}_{\mathrm{m}, \varepsilon}(t) \longrightarrow \mathrm{Bu}_{\varepsilon}(t) \text { strongly in } \mathrm{L}^{\mathbb{F}}\left([0, \infty) ; \mathrm{W}^{*}\right) \quad\left({ }^{\mathrm{V}}>1\right)
$$


and

$$
X_{\varepsilon}(t)=A u_{\varepsilon}(t)+\frac{1}{\varepsilon} B\left(u_{\varepsilon}(t)\right) .
$$

Moreover, with the aid of the inequality

$$
<\beta(u)-\beta(v), u-v>\geqslant\left(\left\|u-p_{k} u\right\|_{v}-\left\|v-p_{K} v\right\|_{v}\right)^{2}
$$

for $u, v \quad v$, we know

$$
\begin{aligned}
\lim _{m \rightarrow \infty}\left\|u_{m, \varepsilon}(t)-p_{k} u_{m}(t)\right\|_{V}= & \left\|u_{\varepsilon}(t)-p_{K} u_{\varepsilon}(t)\right\|_{V} \\
& \text { in } L_{l o c}^{2}([0, \infty)) .
\end{aligned}
$$

The limit function $u_{\varepsilon}(t)$ satisfies

$$
u_{\varepsilon}^{\prime}(t)+A u_{\varepsilon}(t)+B u_{\varepsilon}(t)+\frac{1}{\varepsilon} \beta(u(t))=f(t) \text { a.e. on }[0, \infty)
$$

$$
u_{\varepsilon}(0)=u_{0}
$$

Furthermore, it holds from (32) and (33) that

(32)' $\quad\left\|u_{\varepsilon}(t)\right\|_{V}+\frac{1}{\varepsilon}\left\|u_{\varepsilon}(t)-p_{K} u_{\varepsilon}(t)\right\|_{V}^{2} \leqq c\left(M,\left\|u_{0}\right\|_{V}\right)$

and

$$
\int_{t}^{t+1}\left|u_{\varepsilon}^{\prime}(s)\right|^{2} d s \leqq c\left(M,\left\|u_{0}\right\|_{V}\right)
$$


for $t \geq 0$. Then we may suppose, as $\varepsilon \longrightarrow 0$,

(38)

$$
\begin{aligned}
& u_{\varepsilon}^{\prime}(t) \longrightarrow u_{\varepsilon}^{\prime}(t) \quad \text { weakly in } L_{l o c}^{2}([0, \infty) ; V), \\
& u_{\varepsilon}(t) \longrightarrow u^{\prime}(t) \quad \begin{array}{l}
\text { weakly* in } L^{\infty}([0, \infty) ; V), \\
\text { and in } C_{10 c}([0, \infty) ; H),
\end{array}
\end{aligned}
$$

$$
A u_{\varepsilon}(t) \longrightarrow \dot{X}(t) \text { weakly** in } L^{\infty}\left([0, \infty) ; V^{*}\right)
$$

and

$$
\mathrm{Bu}_{\varepsilon}(t) \longrightarrow \mathrm{Bu}(t) \text { strongly in } \mathrm{L}^{\mathrm{r}}\left([0, \infty) ; \mathrm{W}^{*}\right)\left(V_{\mathrm{r}}>1\right)
$$

Moreover from (32)'

$$
u_{\varepsilon}(t)-p_{K_{\varepsilon}} u_{k}(t) \longrightarrow 0 \text { in } L^{\infty}([0, \infty) ; v),
$$

which implies easily

$$
u(t) \in K \text { a.e. on }[0, \infty) \text {. }
$$

By a standard monotonicity argument (see Biroli [1]) we see $X(t)=A u(t)$ a.e. on $[0, \infty)$, and by $(37)$ we have

$$
<u^{\prime}(t)+A u(t)+B u(t)-f(t), v(t)-u(t)>\geq 0
$$

for $\forall v(t) \in L^{\mathrm{P}}([0, \infty) ; \mathrm{V})$ with $\mathrm{v}(t) \in \mathrm{K}$ a.e. on $[0, \infty)$. We summarize above result in the following

Theorem 2 .

Let $p>\alpha+2$. Then under the assumptions $A_{1}, A_{2}$ and $A_{3}$, the 
problem (1)-(2) admits a solution $u(t)$ such that

$$
\|u(t)\|_{v}+\int_{t}^{t+1}\left|u^{\prime}(s)\right|^{2} d s \leqq c\left(M,\left\|u_{0}\right\|_{V}\right)<\infty
$$

for $\quad t \geq 0$, where we set $M \equiv \sup _{t} \delta(t)$.

Next, we assume $2 \leq p<\alpha+2$. As is already seen, for the existence of solution it suffices to show the boundedness of $u_{m, \varepsilon}(t)$ by a constant independent of $m$ and $\varepsilon$. For this we set further

$$
\tilde{G}_{\varepsilon, 0}(u)=k_{0}\|u\|_{V}^{p}-k_{1} s^{\alpha+2}\|u\|_{V}^{\alpha+2}+\frac{1}{2 \varepsilon}\left\|u-p_{K} u\right\|_{V}^{2}
$$

and

$$
\tilde{G}_{\varepsilon, I}(u)=\tilde{G}_{\varepsilon, 0}(u)+\frac{1}{2 \varepsilon}\left\|u-p_{K} u\right\|_{V}^{2} \text {, }
$$

where $S$ is a constant such tat $\|u\|_{W} \leq S\|u\|_{V}$ for $u \in V$. Note that

$$
G_{\varepsilon, 0}(u) \geqq \tilde{G}_{\varepsilon, 0}(u), G_{\varepsilon, l}(u) \geqq \tilde{G}_{\varepsilon, l}(u) \geqq \tilde{G}_{\varepsilon, 0}(u),
$$

and $G_{\varepsilon, l}(u) \geq G_{\varepsilon, 0}(u)-2 k_{I}\|u\|_{W}^{\alpha+2}$ for $u \in V$. Let us determine $x_{0}>0$ and $D_{0}>0$ as follows.

$$
\max _{x \geq 0}\left(k_{0} x^{p}-k_{3} s^{\alpha+2} x^{\alpha+2}\right)=k_{0} x_{0}^{p}-k_{3} s^{\alpha+2} x^{\alpha+2} \equiv D_{0} .
$$

Then 'the stable set' $\mathcal{W}$ is defined by 


$$
W=\left\{u \in V \mid G_{\varepsilon, I}(u)<D_{0} \text { and }\|u\|_{v}<x_{0}\right\}
$$

Let us assume the initial value $u_{0} \in W \cap K$, and let $M<m_{0}^{\prime} \equiv$

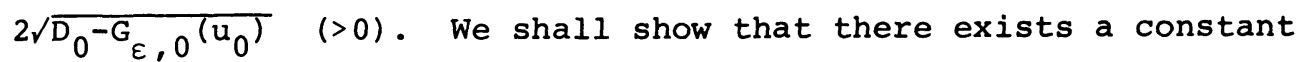
$M_{0}>0$ such tht if $M_{0} M_{0}, u_{m, \varepsilon}(t) \in W$ for $t \leq t_{m}$ provided that $m$ is sufficiently large. First, by (29),

$$
G_{\varepsilon, 0}\left(u_{m, \varepsilon}(t)\right) \leqq G_{\varepsilon, 0}\left(u_{0}\right)+\frac{1}{4} M+\eta<D_{0}
$$

if $0 \leq t \leq \min \left(1, t_{m}\right)$, for sufficiently small $\eta>0$ and large $m$. The inequality (43) implies $t_{m}>1$. Thus, if our assertion were false, there would exist a time $E>I$ such that

$$
G_{\varepsilon, 0}\left(u_{m, \varepsilon}(t)\right)<D_{0} \text { if } \quad 0 \leq t<\bar{t}
$$

and

$$
G_{\varepsilon, 0}\left(u_{m, \varepsilon}(\bar{t})\right)=D_{0}
$$

By (28) with $t_{2}=\bar{t}, t_{1}=\bar{t}-1$ we have easily

$$
\int_{\bar{t}-1}^{\bar{t}}\left|u_{m, \varepsilon}^{\prime}(s)\right|^{2} d s \leqq M^{2}
$$

and hence

$$
\begin{aligned}
\int_{\bar{t}-1}^{\bar{t}} G_{\varepsilon, 1}\left(u_{m, \varepsilon}(s)\right) d s & \leq \int_{\bar{t}-1}^{\bar{t}}\left|-u_{m, \varepsilon}^{\prime}(s)+f(s)\right|\left|u_{m, \varepsilon}(s)\right| d s \\
& \leq 2 M S_{1} x_{0}
\end{aligned}
$$


where $S_{1}$ is a constant such that

$$
\|\mathrm{u}\|_{\mathrm{H}} \leqq \mathrm{s}_{1}\|\mathrm{u}\|_{\mathrm{V}} \quad \text { for } \quad \mathrm{u} \in \mathrm{V} \text {. }
$$

Therefore, if we assume $M<M_{0}^{\prime \prime} \equiv D_{0} / 2 S_{1} x_{0}$, there exists a time $t$ * $\epsilon[\bar{t}-1, \bar{t}]$ such that

$$
G_{\varepsilon, I}\left(u_{m, \varepsilon}\left(t^{*}\right)\right) \leqq 2 M S_{1} x_{0} \text { and }\left\|u_{m, \varepsilon}\left(t^{*}\right)\right\|_{V} \leq x(M)
$$

where $x(M) \quad\left(<x_{0}\right)$ is the smaller root of the numerical equation

$$
k_{0} x^{p}-k_{1} s^{\alpha+2} x^{\alpha+2}=2 M S_{1} x_{0} \quad\left(<D_{0}\right)
$$

We use again (28) to obtain

$$
G_{\varepsilon, 0}\left(u_{m, \varepsilon}(\bar{t})\right) \leqq G_{\varepsilon, 0}\left(u_{m, \varepsilon}\left(t^{*}\right)\right)+\frac{1}{4} M^{2}
$$

$$
\begin{aligned}
& \leq G_{\varepsilon, 1}\left(u_{m, \varepsilon}\left(t^{*}\right)\right)+\frac{1}{4} M^{2}+2 k_{1} s^{\alpha+2}\left\|u_{m,}\left(t^{*}\right)\right\|_{V}^{\alpha+2} \\
& \leq 2 M S_{1} x_{0}+\frac{1}{4} M^{2}+2 k_{1} s^{\alpha+2} x(M)^{\alpha+2} .
\end{aligned}
$$

Now we determine ${ }_{0}^{m}>0$ as the largest number such that

$$
2 k_{1} S^{\alpha+2} x(M " ')+2 M^{\prime \prime} S_{1} x_{0}+\frac{1}{4} M^{\prime \prime \prime} 2=D_{0}\left(M_{0}^{\prime \prime \prime} \leqq M_{0}^{\prime \prime}\right)
$$

and set $M_{0} \equiv \min \left(M_{0}^{\prime}, M_{0}^{\prime \prime \prime}\right)$. Then, assuming $M<M_{0}$, we have by (51)

$$
G_{\varepsilon, 0}\left(u_{m, \varepsilon}(\bar{t})\right)<D_{0}
$$


which contradicts to (45). Consequently, if $M_{K} M_{0}, u_{m, \varepsilon}(t)$ exists on $[0, \infty)$ for large $m$ and it holds that

$$
\begin{aligned}
& \left\|u_{m, \varepsilon}(t)\right\|_{v}<x_{0}, \int_{t}^{t+1}\left|u_{m, \varepsilon}^{\prime}(s)\right|^{2} d s \leqq \text { const. }<\infty \\
& \text { and } \\
& G_{\varepsilon, 0}\left(u_{m, \varepsilon}(t)\right)<D_{0} \text { for } t \in[0, \infty) .
\end{aligned}
$$

Thus, applying the monotonicity and compactness arguments, we obtain the following

Theorem 3.

Let $2 \leq p<\alpha+2$ and $M<M_{0}$. Then the problem $(1)-(2)$ admits a solution $\underline{u}$ satisfying

$$
\|u(t)\|_{v} \leqq x_{0} \quad \text { and } \quad \int_{t}^{t+1}\left|u_{m, \varepsilon}^{\prime}(s)\right|^{2} \text { ds } \leqq \text { const. }<\infty \text {. }
$$

Moreover, we note that the approximate solutions $u_{m, \varepsilon}(t)$ (m: large) satisfy

$$
\begin{aligned}
G_{\varepsilon, 0}\left(u_{m, \varepsilon}(t)\right) \geqq \tilde{G}_{\varepsilon, 0}\left(u_{m, \varepsilon}(t)\right) \\
\geqq\left(k_{0}-k_{1} s^{\alpha+2} x_{0}^{(\alpha+2)-p)}\left\|u_{m, \varepsilon}(t)\right\|_{V}^{p}\right. \\
\quad+\frac{1}{2 \varepsilon}\left\|u-p_{K} u\right\|_{V}^{2}
\end{aligned}
$$

with $\left(k_{0}-k_{1} s^{\alpha+2} x_{0}^{(\alpha+2)-p}\right)>0$. Therefore the same argument as in the section 2 yields the following 
Theorem 4 .

Let $2 \leqq p<\alpha+2$ and $M<M_{0}$. Then the solution in Theorem 3 satisfies the decay property :

(i) If $p>2$ and $\lim _{t \rightarrow \infty} \delta(t) t(p-1) /(p-2)=0$, then

$$
\|u(t)\|_{V} \leqq c\left(\left\|u_{0}\right\|_{V}\right)(1+t)^{-1 /(p-2)}
$$

or

(ii) If $p=2$ and $\delta(t) \leqq C \exp \{-\lambda t\} \quad(C, \lambda>0)$, then

$$
\|u(t)\|_{V} \leqq C^{\prime} \exp \left\{-\lambda^{\prime} t\right\}
$$

for some $C^{\prime}, \lambda^{\prime}>0$.

Remark. In [3], Ishii proved that $|u(t)| \leq C(1+t)^{-1 /(p-2)}$ if $p>2$ and $|u(t)| \leq C \exp \{-\lambda t\} \quad(c, \lambda>0)$ if $p=2$ for the case $f \equiv 0$. It is clear that our result is much better, because the norm $\|\cdot\|_{\mathrm{V}}$ is essentially stronger than the norm $|\cdot|$.

4. An example

Here we give an typical example. Let $\Omega$ be a bounded domain in $\mathrm{R}^{\mathrm{n}}$ and set

$$
\mathrm{V} \equiv \mathrm{W}_{0}^{1,} \mathrm{P}(\Omega), \mathrm{H}=\mathrm{L}^{2}(\Omega) \quad \text { and } \quad \mathrm{W}=\mathrm{L}^{\alpha+2}(\Omega)
$$

with $0<\alpha<p n /(n-1)+2$ if $n \geqq p+1$ and $0<\alpha<\infty$ if $n \leqq p$. We define $\mathrm{A} ; \mathrm{V} \rightarrow \mathrm{V}^{*}$ by 


$$
<A u, v>=\int_{\Omega} \sum_{i=1}^{n}\left|\frac{\partial u}{\partial x_{i}}\right|^{p-2} \frac{\partial u}{\partial x_{i}} \frac{\partial u}{\partial x_{i}} d x \quad(p \geq 2)
$$

for $u, v \in W_{0}^{1, P}(\Omega)$, and $B: W \rightarrow w^{*}$ by

$$
\mathrm{Bu}=\mathrm{d}(\mathrm{x})|\mathrm{u}|^{\alpha} \mathrm{u} \quad \text { for } \quad \mathrm{u} \in \mathrm{L}^{\alpha+2}(\Omega)
$$

where $d(x)$ is a bounded measurable function on $\Omega$. Moreover we set

$$
\mathrm{k}=\left\{\mathrm{u} \in \mathrm{w}_{0}^{1, p}(\Omega) \mid \mathrm{b}(\mathrm{x}) \leqq \mathrm{u}(\mathrm{x}) \leqq \mathrm{a}(\mathrm{x}) \text { a.e. on } \Omega\right\}
$$

where $a, b$ are measurable function on $\Omega$ with $a(x) \stackrel{\geq 0}{=} b(x)$. Then all the assumptions $A_{1}{ }^{-A_{2}}$ are satisfied. The problem (1) -(2) is equivalent in this case to the problem

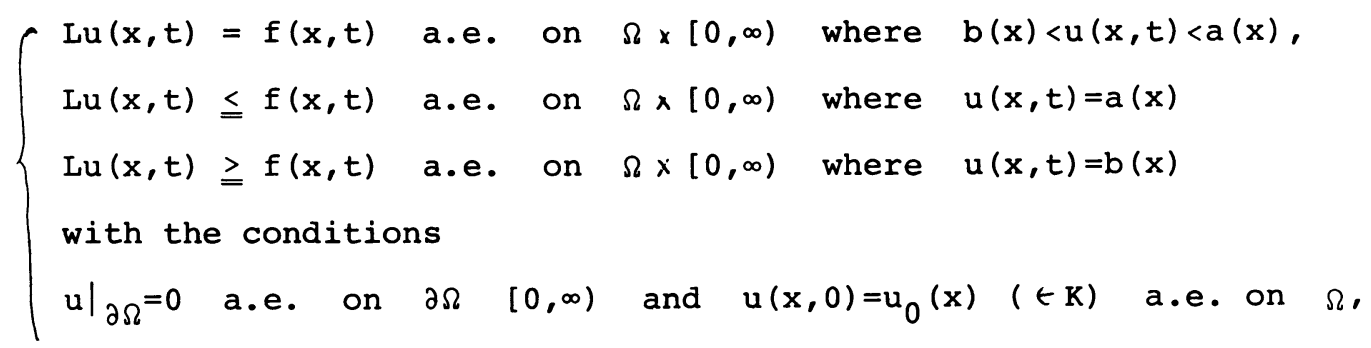

where

$$
L u=\frac{\partial u}{\partial t}-\sum_{j=1}^{n} \frac{\partial}{\partial x_{i}}\left(\left|\frac{\partial u}{\partial x_{i}}\right|^{p-2} \frac{\partial}{\partial x_{i}} u\right)+d(x)|u|^{\alpha} u
$$




\section{REFERENCES}

1. Biroli, M. Sur les inéquations paraboliques avec convexe dépendant du temps: solution forte et solution faible, Riv. Mat. Univ. Parma (3) 3(1974), $33-72$.

2. Brezis, H. Problemes unilateraux, J. Math. Pures appl., 51 (1972), 1-168.

3. Ishif, H. Asymptotic stability and blowing up of solutions of some nonlinear equation, J. Differential Equations, Vol. 26, No. 2 (1977), 291-319.

4. Kenmochi, N. Some nonlinear parabolic variational inequatities, Israel J Math. $\underline{22}$ (1975), 304-331.

5. Lions, J. L. Quelques Méthodes de Résolution des Problemes aux Limites Non Linearies, Dunod, Paris, 1969.

6. Martin, Jr., R. H. Nonlinear Operators and Differential Equations in Banach Spaces, J. Wiley \& sons, Inc. New York, 1976:

7. Nakao, M. On boundedness, perlodicity and almost periodicity of solutions of some nonlinear parabolic equations, J. Differential Equations, 19 (1975), 371-385.

8. Nakao, M. On the existence of bounded solution for nonlinear evolution equation of parabolic type, Math. Rep. College Gen. Educ., Kyushu Univ., XI (1977), 3-14.

9. Nakao, M. Convergence of solutions of the wave equation with a nonlinear dissipative term to the steady state, Mem. Fac. Sc1. Kyushu Univ., 30 (1976), 257-265.

10. Nakao, M. Decay of solutions of some nonlinear evolution equations, J. Math. Ana1. App1. 60 (1977), 542-549.

11. Nakao, M. \& T. Narazaki. Existence and decay of solutions of some nonlinear wave equations in noncylindrical domains, Math. Rep. College Gen. Educ. Kyushu Univ. XI (1978), 117-125.

12. Otani, M. On the existence of strong solutions for $\frac{d u}{d t}(t)+\partial \psi^{1}(u(t))$ $-\partial \phi^{2}(u(t)) f(t)$, J. Fac. Sci. Univ. Tokyo, 24 (1977), 575-605.

13. Yamada, Y. On evolution equations generated by subdifferential operators, J. Fac. Sci. Univ. Tokyo, 23 (1976), 491-515. 


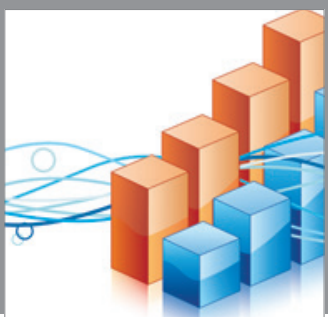

Advances in

Operations Research

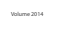

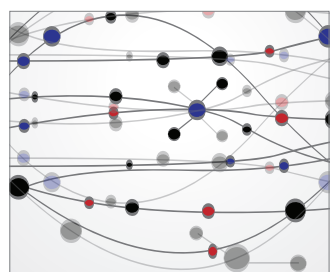

\section{The Scientific} World Journal
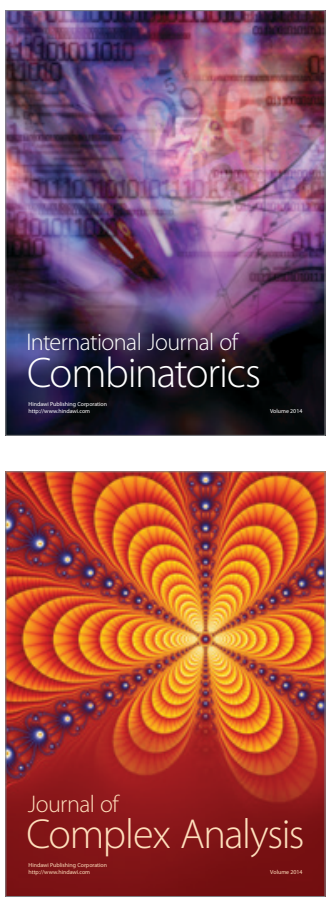

International Journal of

Mathematics and

Mathematical

Sciences
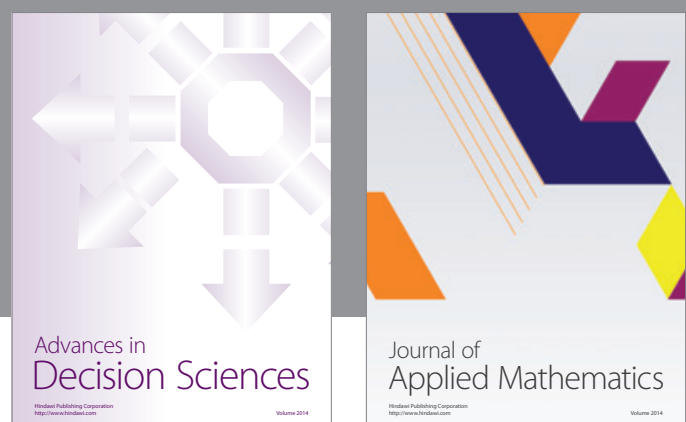

Journal of

Applied Mathematics
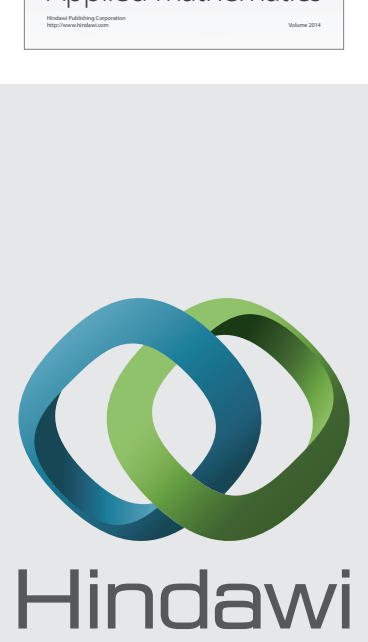

Submit your manuscripts at http://www.hindawi.com
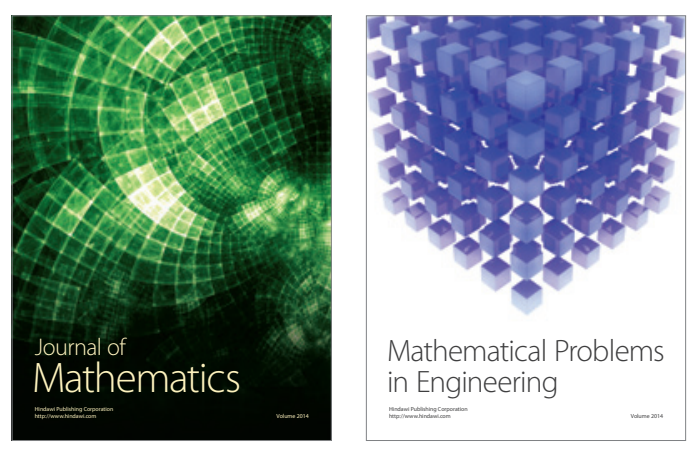

Mathematical Problems in Engineering
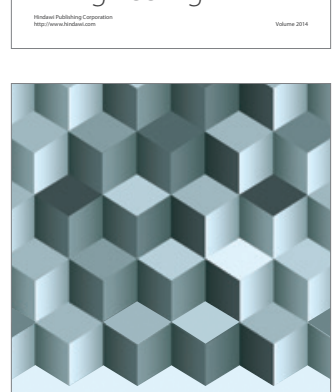

Journal of

Function Spaces
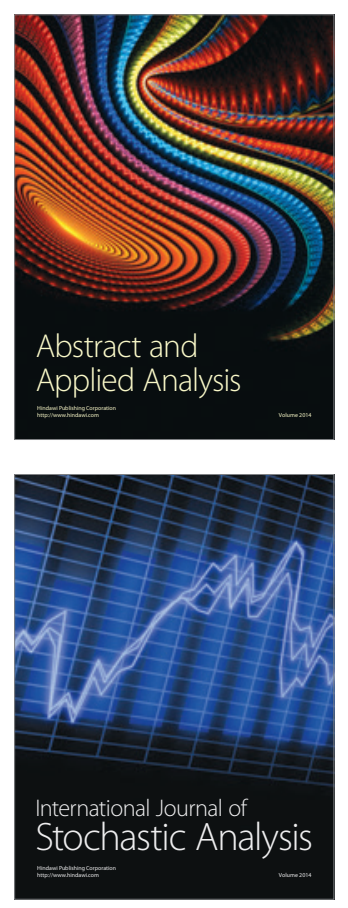

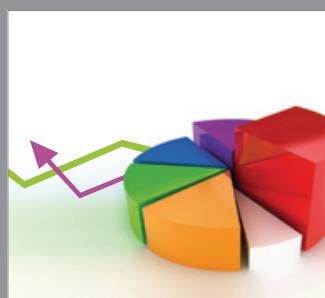

ournal of

Probability and Statistics

Promensencen
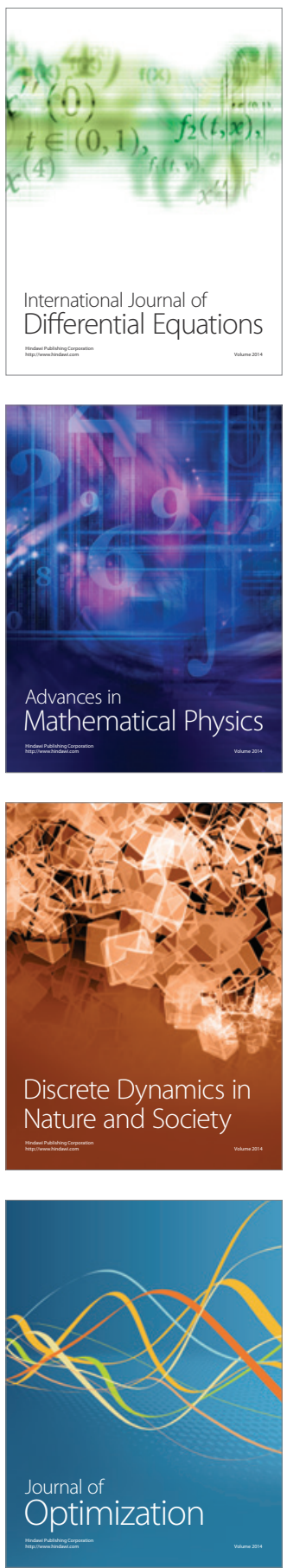